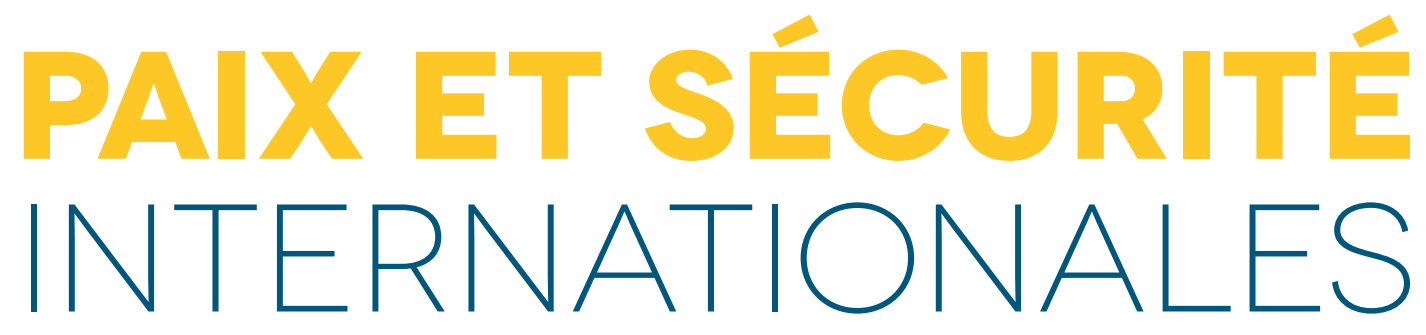

\title{
01
}

2013

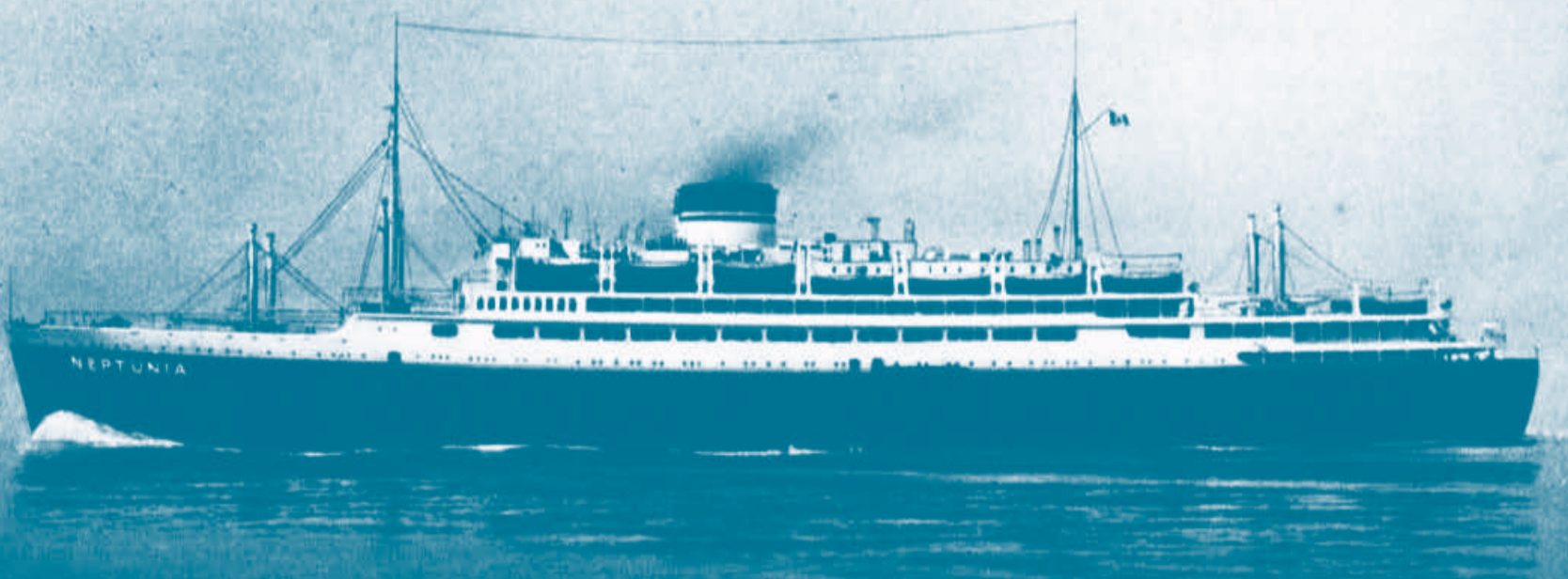

REVUE MAROCO-ESPAGNOLE DE DROIT INTERNATIONAL ET RELATIONS INTERNATIONALES

(NOUVELLE ÉPOQUE) 


\title{
PLACE DU DROIT INTERNATIONAL DANS LE SYSTEME JURIDIQUE DES ETATS
}

Nora SEDDIKI ${ }^{1}$

\begin{abstract}
I. COMPORTEMENT D'HOSTILITÉ. II.- COMPORTEMENT D'APPARENTE FAVEUR. III.- COMPORTEMENT DE RÉELLE FAVEUR. IV.- EN CONCLUSION.
\end{abstract}

\section{PLACE DU DROIT INTERNATIONAL DANS LE SYSTEME JURIDIQUE DES ETATS}

RESUME: La prise en compte du droit international dans le système juridique national des Etats, ne cesse de progresser. C'est dans cette logique que la société internationale s'est précipité afin de construire un arsenal juridique supranational, capable d'harmoniser la divergence accrue qui marquent les systèmes juridiques des Etats . Certes, la place accordée aux conventions internationales n'est pas déterminée par le seul fait de la ratification ou non de celles-ci mais bien plutôt par l'appréciation du système juridique dans sa globalité. Néanmoins, l'effectivité de la norme internationale reste problématique car il est souvent considéré qu'elle est susceptible de générer une perte de souveraineté et de maîtrise du développement économique et social des pays.

MOTS-CLES: Droit international ; Droit Interne ; Ordonnancement juridique ; Economie ; Convention ; Conflit juridique.

\section{EL DERECHO INTERNACIONAL EN EL ORDENAMIENTO JURÍDICO DE LOS ESTADOS}

RESUMEN: La consideración del derecho internacional en el ordenamiento jurídico interno del Estado, continúa creciendo. En este sentido, la comunidad internacional se ha lanzado en el proyecto de construir un arsenal jurídico supranacional, capaz de armonizar la creciente divergencia contenida en los sistemas jurídicos de los Estados. Ciertamente, la importancia dada a las convenciones internacionales no se determina por el mero hecho de su ratificación o no de los mismos, sino más bien por el reconocimiento del sistema jurídico en su conjunto. Sin embargo, la eficacia de la norma internacional es problemática, ya que a menudo se considera que es probable que genere una pérdida de la soberanía y el control del desarrollo económico y social de los países.

PALABRAS CLAVES: Derecho internacional; Derecho interno; Ordenamiento Jurídico; Economía; Convenio; conflicto jurídico.

\section{PLACE OF INTERNATIONAL LAW IN THE LEGAL SYSTEM OF STATS}

ABSTRACT: Consideration of international law in the national legal system of the State, continues to grow. In this sense, the international community rushed to build a supranational legal arsenal, capable of harmonizing the increased divergence contained in the legal systems of States. Certainly, the importance given to international conventions is not determined by the mere fact of its ratification or non thereof but rather the appreciation of the legal system as a whole. However, the effectiveness of the international standard is problematic because it is often considered that it is likely to generate a loss of sovereignty and control of economic and social development of countries.

\footnotetext{
${ }^{1}$ Professeure de Droit des Affaires Internationales à ENCG Tanger, Université Abdelmalek Essaadi.
} 
KEY WORDS: International Law; Internal Law; Legal Order; Economy; Convention; legal conflict.

Dans la quasi-totalité des pays, la prise en compte du droit international dans le système juridique national ne cesse de progresser, et ce, dans de nombreux domaines. Les rapports internationaux aussi bien de droit public que de droit privé ont amené très tôt à l'élaboration des normes internationales afin de contourner l'obstacle de la diversité des systèmes juridiques nationaux. En effet, il existe autant de systèmes juridiques que de pays dans le monde et l'on comptabilise deux cent vingt sept Etats souverains dans le monde.

Dans le souci d'introduire plus de sécurité dans les rapports entre Etats, entre personnes privées (particuliers, entreprises, associations, syndicats) et entre Etats et personnes privées dans un contexte international, les organisations internationales se sont attelées à favoriser la multiplication d'accords internationaux et plus précisément, de Traités internationaux ${ }^{2}$. Ce seul objectif de sécurité rend nécessaire l'adoption d'un corps de règles d'envergure internationale qui est censé s'imposer aux Etats ${ }^{3}$ en venant intégrer progressivement l'ordonnancement juridique interne.

Cet article s'inscrit dans une perspective de développement croissant du droit international dans les pays. Elle se positionne dans un contexte de production scientifique du droit essentiellement caractérisé par une forte charge théorique. Notre cadre de recherche n'est pas circonscrit à un espace géographique limité et il s'attache majoritairement au domaine de l'économie et à l'investissement. Nous nous focaliserons sur les types de comportement possibles pouvant être adoptés par les Etats par rapport à la reconnaissance et à l'effectivité de la norme internationale. Il sera envisagé trois types de comportement marquant une hostilité affichée (I), une apparence de faveur (II) et une réelle faveur pour l'admission d'une telle norme (III).

\section{COMPORTEMENT D'HOSTILITÉ}

La position d'hostilité se manifeste par rapport à l'acceptation et la réception de la norme internationale dans le système normatif des Etats. Cette norme internationale adopte la forme d'une convention internationale et des principes généraux du droit international. Les conventions concernées par cette attitude sont essentiellement celles d'ordre multilatéral

\footnotetext{
${ }^{2}$ D'autant que ne serait-ce qu'en matière d'affaires internationales celles-ci tiennent une place de plus en plus importante dans le monde de l'entreprise.

${ }^{3}$ AUDIT, B., " Le Droit international privé en quête d'universalité. Cours général », Recueil des cours, tome 305, 2003 ; TEUBNER, G., "Un droit spontané dans la société mondiale ? », in Le droit saisi par la mondialisation, sous la dir. De CH.-A MORAND, Bruylant, Bruxelles, 2001.
} 
et parfois bilatéral ${ }^{4}$. Tandis que les principes rejetés sont le plus souvent les vecteurs d'une idéologie libérale prônant la loi du marché. Le principe philosophique d'autonomie de la volonté et ses pendants pratiques à savoir la liberté contractuelle et la force obligatoire des contrats sont les instruments juridiques au service de cette idéologie.

Le positionnement juridique d'un Etat au regard de ces organisations internationales ${ }^{5}$ et également des investisseurs étrangers est, pour partie, lié au nombre de ratification des conventions internationales et à la place accordée à cette source de droit international dans le système juridique et judiciaire des Etats. Certains auteurs iront jusqu'à défendre la thèse du retrait de l'Etat au profit d'instances supranationales, vecteur d'un « nouvel ordre juridique économique » et d'une nouvelle forme de « police des marchés » tandis que d'autres refusent d'adhérer à cette idée.

Il est vrai que l'adoption des sources internationales pose la question de la mise en conformité de la législation des pays par rapport aux standards internationaux. Les organisations internationales mettent progressivement en place un cadre juridique international ${ }^{6}$ qui sert de référent aux Etats comme par exemple les lois modèles de la Commission des Nations Unies pour le droit commercial international (CNUDCI) ou encore celles d'Unidroit.

Les organisations internationales veillent à la mise en conformité des ordonnancements juridiques des Etats. L’Assemblée générale de l'ONU évoque d'ailleurs dans une de ses résolutions : " la coopération entre les Etats en matière de commerce international peut beaucoup contribuer à favoriser les relations amicales et, par conséquent, le maintien de la paix et de la sécurité, rappelant qu'elle estime qu'il est de l'intérêt de tous les peuples, et en particulier de celui des pays en voie de développement, d'améliorer les conditions favorisant un large développement du commerce international. Réaffirmant sa conviction que les divergences entre les lois des divers Etats sur des questions relatives au commerce international constituent un des obstacles au développement du commerce mondial $»^{7}$. De part leurs engagements

\footnotetext{
${ }^{4}$ Notamment en matière de transfert des droits sociaux pour les migrants qui souhaiteraient retourner à titre provisoire voire définitif dans leur pays d'origine.

${ }^{5}$ Le terme est large car il renvoie aussi bien aux organisations internationales qu'aux ONG. Les Organisations internationales, telles celles qui ont été instituées par un traité, lui-même conclu entre des Etats. Ce type d'organisation se distingue fondamentalement des ONG qui ne sont pas instituées par un accord entre Etats mais procèdent d'une initiative privée. Les OI sont des sujets de droit international alors que les ONG ne le sont pas.

${ }^{6}$ Quelles sont les sources du droit international ? La typologie connue et la plus communément admise des sources du droit international est notifiée à l'article 38 du statut de la Cour internationale de justice qui constitue l'institution judiciaire principale des Nations unies. Le statut figure en annexe à la Charte des Nations unies et tout Etat partie à la Charte est ipso facto partie au statut, même s'il fait le choix de ne pas se soumettre à la juridiction de la Cour. On distingue donc généralement dans la logique de l'article 38 : les traités et accords internationaux multilatéraux et bilatéraux, la coutume internationale, les principes généraux du droit, les décisions judiciaires, et à titre subsidiaire la doctrine des publicistes les plus qualifiés des différentes nations, Certains actes émanant des OI.

${ }^{7}$ La résolution 2205 (XXI) de l'Assemblée générale des Nations Unies portant création de la CNUDCI, adoptée sur la base des rapports de la $8^{\text {ème }}$ Commission. Point 88 de l'ordre du jour, documents A/6396 et Add. 1 et 2 .
} 
internationaux les Etats ne peuvent s'y soustraire, et cela ce traduit par une modification de leur législation. D'autant plus que cette évolution témoigne de leur engagement dans le processus de libéralisation des échanges commerciaux. C'est la marque même d'un positionnement juridique attendu des instances internationales et des opérateurs économiques.

Les Etats auraient donc une sorte d'obligation de résultat quant à cette mise en conformité de leur législation. Ils se doivent de suivre le mouvement pour permettre leur développement économique de plus en plus dépendant des marchés extérieurs et par la même des relations d'affaires internationales. La qualification de loi moderne est attribuée à la législation qui se conforme à cette logique d'uniformisation du droit à l'échelle mondiale.

Très peu d'Etats adoptent un comportement ouvertement défavorable au droit international et à sa réception au niveau national car cela suscite méfiance et réprobation de la part de la communauté internationale. Le respect de l'ordre établi s'impose afin que les relations internationales puissent s'établir et prospérer sans subir de perturbations en raison d'un droit national restrictif. Il est donc certain qu'afficher une défaveur induit un jugement critique de la part des défenseurs

Lorsqu'un tel comportement apparait, il peut être justifié par le fait que la recherche de l'uniformité des règles pour satisfaire les impératifs de développement des échanges commerciaux est profitable au système économique mondial mais qu'elle n'est pas totalement satisfaisante pour les Etats qui souhaitent affirmer la spécificité de leur culture juridique et religieuse. Il est vrai que l'uniformité n'a pas que des avantages. Elle impose et elle exclut. Elle « commande » de respecter un corps de règles bien établi et exclut en conséquence toute prise en compte de spécificités.

Bien qu'il s'agisse d'exclure la diversité dans un but d'efficacité, peut-on tout de même considérer que ces pays hostiles à l'uniformité du droit manifestent un refus quant à l'admission systématique d'une certaine conception du droit et l'effacement d'une conception propre? Est-ce que les défaillances de la loi mais également des institutions judiciaires ainsi que le comportement des institutions extrajudiciaires (chambres de commerce, ministères, organismes patronaux...) peuvent être considérés comme la manifestation d'une résistance ? Dans quelle mesure ces défaillances nuisent-elles à l'efficacité générale du droit international ou alors peut-on considérer que ces résistances peuvent être dépassées ?

Ces questions comportent de très importants enjeux pratiques dans ce contexte d'uniformisation sans cesse croissant du droit à l'échelle mondiale. Alors comment se manifeste concrètement ce comportement d'hostilité et cette résistance? 


\section{LE REFUS DE RATIFIER LES PRINCIPALES CONVENTIONS INTERNATIONALES}

Ce sont d'abord les conventions internationales qui participent à l'effacement des incompatibilités entre les droits nationaux et à l'adaptation des législations nationales aux exigences des rapports internationaux. Elles favorisent ainsi la modernisation du droit et concourt au mouvement d'unification et d'harmonisation du droit international mais avec le risque de considérer qu'il s'agit d'un abandon plus ou moins important de souveraineté. D'autant que certaines conventions sont créatrices de nouvelles règles de droit qui sont censées se substituer aux dispositions du droit interne des Etats. Certains Etats refusent de se prêter à un tel relâchement de la force souveraine nationale.

Il semble évident que la ratification des grandes conventions internationales représente un des premiers indicateurs juridiques permettant d'apprécier le système normatif d'un Etat donné. De sorte que le résultat de cette appréciation a des effets directs sur la capacité d'attraction ou de répulsion des investissements directs étrangers. Car il ne faut pas occulter le fait que des classements sont établis par rapport à la viabilité du système juridique de chaque Etat. On pourra citer dans ce sens le célèbre rapport « Doing business » de la Banque mondiale qui depuis 2004 classe tous les ans les 175 systèmes juridiques mondiaux.

\section{DIFFICULTÉ À ADHERER AUX GRANDES ORGANISATIONS INTERNATIONALES}

Cette difficulté peut être le fait d'une volonté manifeste de ne pas se soumettre aux dictats de ces organisations a-nationales. Mais cela peut aussi apparaître comme une forme de sanction en raison des exigences d'admission imposées par ces mêmes organisations. A l'instar de l'OMC ${ }^{8}$, considéré comme l'organisation internationale la plus puissante dans le monde, qui a suspendu pendant de nombreuses années l'adhésion de la Chine, de la Russie et également de l'Algérie.

Cuba et la Corée du Nord sont les pays qui adoptent le comportement le plus hostile à l'égard de l'adhésion aux OI promotrices du développement du commerce international et à la ratification des conventions internationales. Il est notamment reproché de promouvoir une mondialisation discriminatoire de l'économie et la dérèglementation excessive du commerce. Les traités sont considérés comme protecteurs des intérêts des opérateurs économiques des pays les plus développés, dont les multinationales, au détriment des salariés et des pays beaucoup moins développés.

\footnotetext{
${ }^{8}$ Sa mission principale consiste à concevoir un nombre important d'accords afin de supprimer les droits de douane entre les pays et à la lutte contre le protectionnisme douanier.
} 


\section{COMPORTEMENT D'APPARENTE FAVEUR}

Les Etats qui se prêtent à ce type de comportement ont compris qu'ils se trouvent sous l'observation des OI. C'est pour cette raison que face à ce constat et dans le souci permanent de ne pas freiner le flux des investissements étrangers de même que les prêts accordés par les institutions financières internationales, voire l'attribution de fonds d'aide au développement, ces pays se dirigent vers l'adoption d'une telle conduite.

La résistance n’apparait donc pas par le refus d'adhérer aux conventions et organisations internationales. Celle-ci, plus nuancée, sera décelée par la place et l'effectivité accordées à la norme internationale dans le système juridique et judiciaire des Etats concernés.

\section{UNE PLACE SANS GRANDE PORTÉE PRATIQUE}

Dans un premier temps cela signifie que la norme internationale a été ratifiée par le pays. Mais encore faut-il se demander quelle est la valeur juridique de cette ratification?'. Peut-on considérer que la convention internationale est reconnue en tant que source du droit dans l'ordonnancement juridique ${ }^{10}$ ? Car certains pays, tel le Royaume uni, considèrent que la convention est obligatoire et donc effective non pas à partir de la date de sa ratification (pas d'effet direct) mais plutôt dès lors que le Parlement a voté une loi pour l'admission du traité dans le droit du pays. Cela signifie que la norme internationale n'est pas reconnue en tant que telle et quelle doit subir transformation, en loi nationale, pour qu'elle puisse offrir toute son effectivité. Il pourrait arriver que de nombreuses années s'écoulent avant que la convention ratifiée puisse être admise par le biais de la promulgation d'une loi.

Il existe une autre façon de minimiser la valeur de la norme internationale, au regard de la hiérarchie des sources ${ }^{11}$, c'est celle de considérer que la loi nationale a une autorité supérieure à la norme internationale. De sorte que la contradiction des deux textes sur une même

\footnotetext{
${ }^{9} \mathrm{La}$ doctrine se focalise souvent en priorité sur la Constitution pour considérer qu'un pays est favorable ou non au développement du droit international porteur de règles à vocation expansionniste. Cette doctrine n'est pas simplement celle attachée au domaine du droit international public puisque les chercheurs en droit international privé procèdent de même. Les observateurs internationaux (ONG et Organisations internationales) vont aussi dans le même sens.

${ }^{10}$ La classification des systèmes juridiques nationaux, du point de vue des rapports entre les droits d'origine externe et le droit national, est souvent réalisée à partir de deux grands modèles théoriques, censés rendre compte d'une approche d'ensemble des rapports entre droit international et droit interne : les systèmes nationaux sont ainsi fréquemment rattachés à un modèle moniste ou à un modèle dualiste. Chacun de ces modèles renvoie luimême à une conception des liens supposés exister ou ne pas exister entre la sphère du droit international et la sphère du droit interne : le modèle dualiste est fondé sur une séparation, autrement dit une dualité, entre ces deux ensembles, tandis que le modèle moniste retient une conception unitaire ou unificatrice de ceux-ci.

${ }^{11}$ La notion de hiérarchie renvoie à l'organisation d'un ensemble en une série où chaque terme est supérieur au terme suivant, par un caractère de nature normative.

C'est donc l'idée de l'autorité supérieure d'une norme par rapport à une autre qui, en droit, constitue la hiérarchie des normes juridiques. Les termes les plus souvent évoqué sont ceux de suprématie ou de primauté d'une norme sur l'autre.
} 
thématique amène à privilégier la norme nationale. De même que l'absence de reconnaissance expresse du principe peut conduire au même résultat à savoir la prise en compte de la loi au détriment de la convention internationale.

\section{LA RETICENCE DES JUGES NATIONAUX}

Il semble évident qu'il s'agit d'un des aspects essentiels du droit international et de sa réelle effectivité. Le comportement des tribunaux face à l'application de la norme internationale apparait par le fait des décisions de justice. Ces décisions peuvent témoigner d'une attitude plus ou moins marquée d'hostilité.

On retrouve cette forme de protectionnisme de la part des juridictions publiques dans de nombreux pays, dont certains affichent pourtant une volonté manifeste de défendre le libéralisme économique et la mise à l'écart de l'Etat et des institutions nationales tels les tribunaux.

\section{COMPORTEMENT DE RÉELLE FAVEUR}

Cela concerne principalement les pays développés et dans une moins grande mesure les pays en cours de développement. Certains de ces pays sont à l'initiative des projets de conventions internationales dans le cadre organisé des OI.

\section{L'INTÉGRATION DES NORMES INTERNATIONALES DANS LE SYSTÈME JURIDIQUE}

Les pays concernés par ce type de comportement adoptent la plupart des conventions internationales et ratifient de nombreuses conventions bilatérales. Ces normes sont directement applicables dans l'Etat sans qu'il soit nécessaire de faire intervenir des normes nationales. Cela signifie que la norme est acceptée en tant que source à part entière de droit intégrée dans la hiérarchie des normes juridiques.

Certains pays ont généralisé le principe mais ont considéré que dès lors que l'applicabilité de la convention internationale ratifiée a des incidences sur les finances publiques de l'Etat, le Parlement doit l'approuver par l'adoption d'une loi. On pourra citer, notamment, le cas de la France et du Maroc qui ont fait de cette règle un principe constitutionnel. Dans le cas de ces pays il ne s'agit certainement pas de neutraliser la norme internationale mais bien plutôt de lui offrir une réelle assise financière.

L'intégration de la norme internationale se fera en considérant que celle-ci a une autorité supérieure à la loi du pays. La Constitution pourra être modifiée dans le sens de l'admission de l'ouverture du droit national au droit international. 


\section{DES JUGES NATIONAUX FAVORABLES A L'EFFECTIVITÉ DU DROIT INTERNATIONAL}

En règle générale les juges ont connaissance des grandes conventions internationales et ils ne se refusent pas à asseoir leurs décisions sur les dispositions de ces conventions quand cela s'avère nécessaire.

L'analyse de la jurisprudence témoigne de la prise en considération du droit international. Il est notamment garanti une interprétation uniforme des règles posées par la convention. Si le sens d'une telle norme est obscur c'est le principe de l'effet utile, c'est-à-dire le respect de l'efficacité, qui prime avant tout, en s'appuyant sur le raisonnement téléologique.

\section{EN CONCLUSION}

La place accordée aux conventions internationales n'est pas déterminée par le seul fait de la ratification ou non de celles-ci mais bien plutôt par l'appréciation du système juridique dans sa globalité. L'effectivité de la norme internationale reste problématique car il est souvent considéré qu'elle est susceptible de générer une perte de souveraineté et de maitrise du développement économique et social des pays. 


\section{PAIX ET SÉCURITÉ INTERNATIONALES}

REVUE MAROCCO-ESPAGNOLE DE DROIT INTERNATIONAL ET RELATIONES INTERNATIONALES

NOUVELLE ÉPOQUE

SUMARIE / Janvier - Décembre 2013 / N 1

\section{ÉDITORIAL}

Rachid el Houdaïgui, Alejandro del Valle Gálvez y Miguel Acosta Sánchez

\section{ÉTUDES}

José Manuel SOBRINO HEREDIA

La politique maritime integré de la UE et les bassins maritimes européens

Antonio BLANC ALTEMIR, Bénédicte REAL

Un nouevel élan dans les relations euro-méditerranéennes

Marcello DI FLLIPPO

Irregular Migration across the Mediterranean Sea: Problematic Issues Concerning the International Rules on

Safeguard of Life at Sea

\section{Zakaria ABOUDDAHAB}

La participation du Maroc au Partenariat de Deauville sur fond the transition dans les pays arabes

Irene FERNÁNDEZ MOLINA, Miguel HERNANDO DE LARRAMENDI

La construction de la interdépendance entre l'Espagne et le Maroc (1995-2009)

Victor L. GUTIIÉRREZ CASTILLO, Juan J. GARCÍA BLESA

Le Détroit de Gibraltar et l'application de la normative de l'Union Europeenne relativa aux énergies renovables

Mohamed ALI TOUZI

La vision de la Méditerranée depues les deux rives: una perpective a débatre

\section{NOTES}

Jean DUFOURCQ

Vers un espace stratégique euromahrébin

Nora SEDDEKI

Place du droit international dans le système juridique des Etats

Carlos ECHEVERRÍA JESÚS

Kidnappings as a terrorist instrument of AIQM and the MUJAO

Youssef EL HAMDOUNI

Internet y la Primavera Árabe: hacia una nueva percepción del ciberespacio

\section{DOCUMENTATION}

